\title{
A Systematic Review of Neoadjuvant Therapy Compared to the "Resection First" Approach for Patients with Borderline Resectable Pancreatic Adenocarcinoma
}

\author{
Paul G. Toomey ${ }^{1}$, Sharona Ross ${ }^{1}$, Shalin Shah ${ }^{2}$, Alexander S. Rosemurgy ${ }^{1}$ \\ ${ }^{1}$ The Southeastern Center for Digestive Disorders \& Pancreatic Cancer, Advanced Minimally Invasive \& Robotic Surgery, Florida \\ Hospital Tampa, Tampa, USA; ${ }^{2}$ Florida Cancer Specialists, Tampa, USA. \\ Email: arosemurgy@hotmail.com
}

Received November $7^{\text {th }}, 2013$; revised December $5^{\text {th }}, 2013$; accepted December $13^{\text {th }}, 2013$

Copyright (c) 2014 Paul G. Toomey et al. This is an open access article distributed under the Creative Commons Attribution License, which permits unrestricted use, distribution, and reproduction in any medium, provided the original work is properly cited. In accordance of the Creative Commons Attribution License all Copyrights (C) 2014 are reserved for SCIRP and the owner of the intellectual property Paul G. Toomey et al. All Copyright @ 2014 are guarded by law and by SCIRP as a guardian.

\section{ABSTRACT}

Background: Survival for patients with pancreatic adenocarcinoma continues to be poor. Patients with pancreatic adenocarcinoma that is deemed borderline resectable have imaging that shows disease involvement of the portal vein and/or superior mesenteric vein that is amenable to reconstruction or abutment $(\leq 180$ degrees) of the superior mesenteric artery. The best initial treatment for patients with borderline resectable pancreatic adenocarcinoma has yet to be determined. Proponents of neoadjuvant therapy purport its utility for patients with borderline resectable pancreatic adenocarcinoma with the intention of increasing the likelihood of a microscopically negative (R0) margin, but the consequences of this approach are not established. This study was undertaken to systematically review the outcomes for patients with borderline resectable pancreatic adenocarcinoma to compare neoadjuvant therapy to a "resection first" approach. Methods: A MEDLINE/PubMed search was undertaken to find all studies regarding patients who underwent neoadjuvant therapy for patients with borderline resectable pancreatic adenocarcinoma. Results: A total of 112 studies were found regarding borderline resectable pancreatic cancer. Fourteen studies contained cohorts of patients with borderline resectable pancreatic adenocarcinoma who received neoadjuvant therapy $(n=471$ patients) or a resection-first approach $(n=76$ patients). Resection after neoadjuvant therapy was undertaken for $233(49 \%)$ patients. Neoadjuvant therapy followed by an R0 resection occurred for $42 \%$ of patients. For patients who underwent resection first, $71 \%(54 / 76)$ had an R0 margin. Conclusion: Patients with borderline resectable pancreatic adenocarcinoma were more often found to undergo neoadjuvant therapy than a "resection first" approach in the available literature. Although neoadjuvant therapy portends a high rate of $\mathrm{RO}$ resections, less than half of the patients who undergo neoadjuvant therapy for borderline resectable pancreatic adenocarcinoma undergo resection. Patients who undergo "resection first" for borderline resectable pancreatic adenocarcinoma have an increased chance for a resection and an Ro margin compared to patients who undergo neoadjuvant therapy for borderline pancreatic adenocarcinoma.

\section{KEYWORDS}

Adenocarcinoma; Borderline; Cancer; Chemotherapy; Chemoradiation; Neoadjuvant; Pancreatic; Resectable

\section{Introduction}

Pancreatic cancer continues to have a dismal prognosis, with four out of five patients initially presented with locally advanced unresectable tumors or metastatic disease that precludes any prospect for complete tumor extirpation [1-3]. Complete tumor resection for pancreatic cancer is the only hope for cure and has been shown to significantly improve survival [4-8]. Even still, patients who undergo pancreatic resection for pancreatic adenocarcinoma portend a 5-year survival of only about $20 \%$ $[9,10]$.

Borderline resectable pancreatic adenocarcinoma is a 
preoperative diagnosis based on imaging that was clearly defined by Varadhachary et al. in 2005 [10,11]. The consensus statement by the Americas Hepato-Pancreato-Biliary Association, Society of Surgical Oncology, Society for Surgery of the Alimentary Tract (AHPBA/SSO/ SSAT) in 2009, the current National Comprehensive Cancer Network (NCCN) definition, and the MD Anderson definitions have all emerged as the leading contenders for one unified definition (Table 1) [10-13]. A main variation among definitions is the level of portovenous involvement, with abutment alone (AHPBA/SSO/SSAT) compared to impingement and/or short segment occlusion (NCCN/MD Anderson) being included as borderline resectable [10-13].

Gemcitabine has become the standard adjuvant chemotherapeutic regimen for patients with pancreatic adenocarcinoma. Approved by the Food and Drug Administration in 1997 for its beneficial effects on pain and quality of life, gemcitabine has less impact on prolonging survival in patients with pancreatic adenocarcinoma $[14,15]$. Gemcitabine was found to significantly improve, but not substantially improve, survival compared to 5-FU in a randomized control trial for patients with locally advanced or metastatic pancreatic cancer (5.6 months, 4.4 months; respectively) [14]. Neoadjuvant gemcitabine for patients presenting with resectable disease led to $82 \%$ of patients ultimately undergoing resection and $89 \%$ of them having R0 (microscopically/macroscopically negative margins) resection [16]. Patients who are presented with non-metastatic, locally advanced, unresectable pancreatic adenocarcinoma and who receive neoadjuvant gemcitabine undergo resection $46 \%$ of the time and $39 \%$ - 60\% undergo an R0 resection [16,17]. Patients with borderline resectable pancreatic adenocarcinoma typically receive neoadjuvant gemcitabine in this modern era with the expectation of increased resectability without vascular reconstruction and an increased chance of R0 resections compared to patients who undergo a "resection first" approach. The consequences of neoadjuvant therapy are not trivial, as a significant portion of patients undergoing neoadjuvant therapy are unable to complete therapy and recover to undergo resection. Patients who undergo restaging during neoadjuvant therapy are also found to have progressive disease with locally advanced, unresectable tumors or metastatic diseases that preclude resection. Resection after neoadjuvant therapy is by no means universal.

This study was undertaken to systematically review the available data on patients with borderline resectable pancreatic adenocarcinoma who undergo neoadjuvant therapy compared to a "resection first" approach for borderline resectable pancreatic adenocarcinoma to determine the best approach for patients with borderline resectable pancreatic adenocarcinoma in terms of resectability, R0 resections, and long-term outcome. We hypothesized that the proportion of patients who ultimately undergo resection would be lower in patients who undergo neoadjuvant therapy than that in patients who underwent

Table 1. Definitions of borderline resectable pancreatic adenocarcinoma.

\begin{tabular}{|c|c|c|c|c|}
\hline & $\begin{array}{l}\text { Portal vein/superior } \\
\text { mesenteric vein }\end{array}$ & $\begin{array}{c}\text { Superior } \\
\text { mesenteric artery }\end{array}$ & $\begin{array}{l}\text { Common hepatic artery/ } \\
\text { gastroduodenal artery/celiac axis }\end{array}$ & Miscellaneous \\
\hline $\begin{array}{c}\text { Varadhachary et al. } \\
\text { Pancreas } 2005 \\
\text { (MD Anderson) }\end{array}$ & Segmental venous occlusion & $\begin{array}{c}\text { Abutment } \\
(<180 \text { degrees })\end{array}$ & $\begin{array}{l}\text { Short segment abutment } \\
\text { ( }<180 \text { degrees) of the } \\
\text { common hepatic artery (typically } \\
\text { at the gastroduodenal artery) }\end{array}$ & Not applicable \\
\hline $\begin{array}{l}\text { Callery et al. } \\
\text { Annals Surg Onc } 2009 \\
\text { (AHPBA/SSAT/SSO } \\
\text { Consensus Statement) }\end{array}$ & $\begin{array}{l}\text { Abutment with or without } \\
\text { impingement and narrowing } \\
\text { or encasement safe } \\
\text { for reconstruction }\end{array}$ & $\begin{array}{c}\text { Abutment } \\
\text { (<180 degrees) }\end{array}$ & $\begin{array}{l}\text { Gastroduodenal artery encasement } \\
\text { up to the hepatic artery with either } \\
\text { short segment encasement or } \\
\text { abutment ( }<180 \text { degrees) } \\
\text { of the common hepatic artery }\end{array}$ & Not applicable \\
\hline
\end{tabular}


an "upfront resection." Additionally, we also suspected that patients who undergo neoadjuvant therapy with subsequent resection would have an increased number of R0 resection and therefore an increased overall survival compared to patients who undergo an "upfront resection.”

\section{Materials and Methods}

A MEDLINE/Pubmed search undertaken with the key words "borderline resectable pancreatic", "borderline resectable pancreas", and "borderline chemotherapy pancreatic" yielded 112 studies (Figure 1). Ninety-one of the studies available were from 2005 to 2012, undoubtedly a consequence of the adoption of the definition for borderline resectable pancreatic adenocarcinoma in 2005. Ninety-eight studies were excluded from analysis, with 14 studies having cohorts of borderline resectable pancreatic adenocarcinoma patients receiving neoadjuvant therapy or resection first. Studies were excluded from analysis if we were unable to separate out the patients with borderline resectable pancreatic adenocarcinoma who were combined in analysis with patients with resectable or locally advanced pancreatic adenocarcinoma. We also excluded studies that did not specify if the patients received radiation alone or in combination with chemotherapy for neoadjuvant therapy. Studies that included only patients with borderline resectable to due to poor performance status or studies that had a non-standard definition for patients with borderline resectable pancreatic adenocarcinoma were excluded. Studies that only included patients with unresectable disease or data not pertinent to patients undergoing neoadjuvant therapy for borderline resectable pancreatic adenocarcinoma were excluded from analysis. Consensus statements, opinion articles, and reviews involving redundant studies were also excluded from analysis. Three of the 14 studies were near duplicate reports from the same institution with overlapping time intervals and therefore two of those studies were excluded. Two additional studies were identified; one from available online ASCO abstracts and
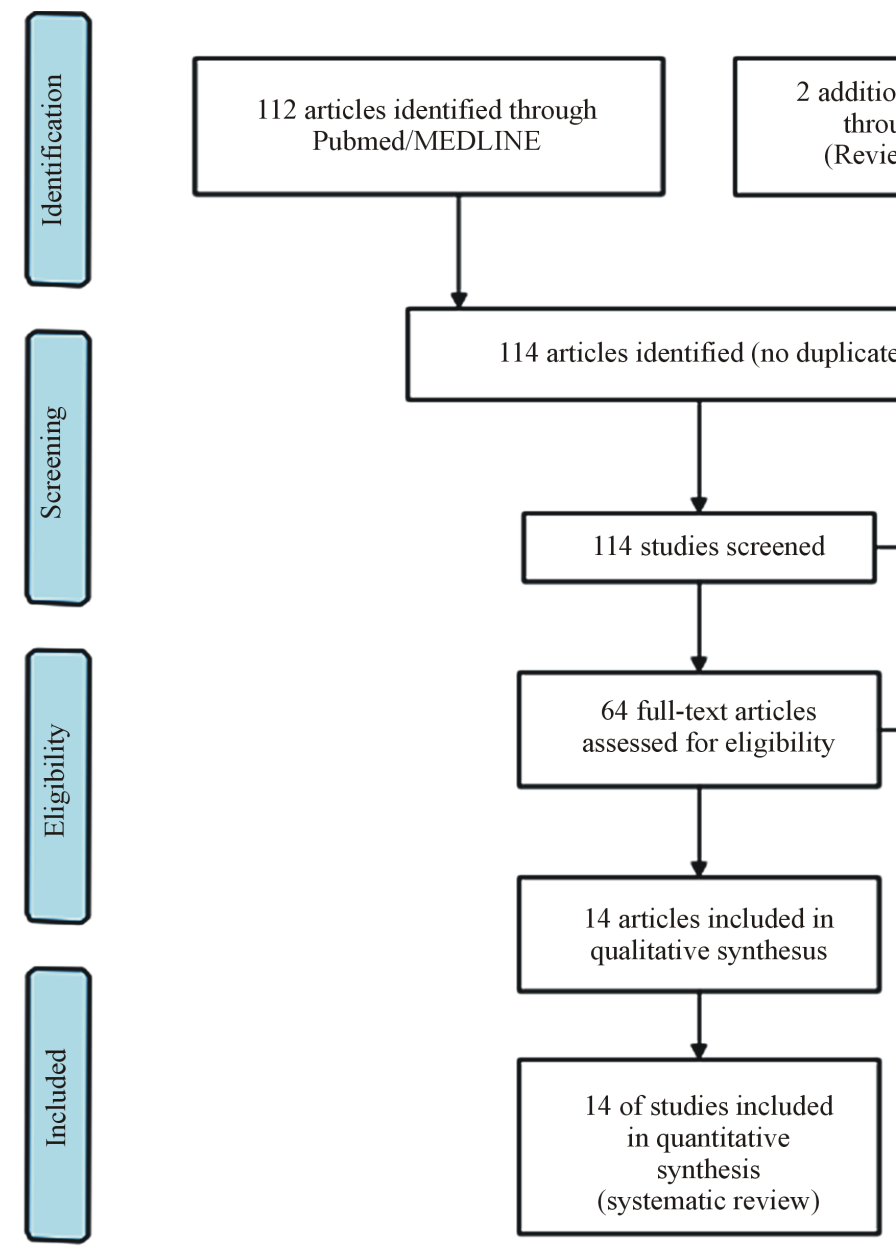

additional articles identified

through other sources

(Review/ASCO abstract)

50 articles removed

(Consensus statements [23],

Reviews [21])

50 articles removed

- Studies from same institution during same time interval; largest series included [2]

- Unable to separate cohort (BLR unresectable, chemo/no chemol), does not specify BLR neoadjuvant therapy, only performance status BLR patients, non-standard BLR definition [14]

- Patients with only unresectable tumors [1]

- No cohort relevant to BLR neoadjuvant, resection, or margin status [33]

Figure 1. Flowchart for studies identified for analysis with a MEDLINE/PubMed search, ASCO abstracts and from available reviews for patients with borderline resectable pancreatic adenocarcinoma (BLR = borderline resectable). 
one from a prior neoadjuvant therapy review. Of the included 14 studies, 10 studies had a cohort of patients who underwent neoadjuvant therapy prior to resection and did not have a cohort including the "resection first" approach. One study had patients who underwent resection first and three studies had both cohorts of patients who either underwent neoadjuvant therapy or resection first.

\section{Results}

A total of 14 studies with borderline resectable pancreatic adenocarcinoma cohorts could be isolated and analyzed: patients either underwent neoadjuvant therapy with the intention for subsequent resection or a "resection first" approach. Of the 14 studies included, ten studies include patients with borderline resectable pancreatic adenocarcinoma who underwent neoadjuvant therapy, one study includes patients who underwent upfront resection and three studies included both cohorts of patients who underwent neoadjuvant therapy or upfront resection. Gemcitabine-based neoadjuvant therapy was the utilized in 14 of the 16 studies with two of the studies excluded from analysis due to overlapping time intervals from the same institution (Table 2).

A total of 471 patients underwent neoadjuvant therapy and 76 patients underwent resection first. After neoadjuvant therapy, 49\% (233/471) of patients underwent resection (Table 3). Ten studies ( $\mathrm{n}=398$ patients) included margin status after neoadjuvant therapy for patients who underwent resection. Patients who underwent resection after neoadjuvant therapy had an R0 (microscopically/ macroscopically negative) margin 91\% (172/190) of the time (Table 4). Patients who underwent upfront resection had an R0 margin 71\% (54/76) of the time (Table 5).

Eight studies ( $\mathrm{n}=385$ patients) included subgroup analysis of patients with borderline resectable pancreatic adenocarcinoma who underwent neoadjuvant therapy and did not undergo resection as well as patients who were resected after neoadjuvant therapy. Patients completed their neoadjuvant therapy $90 \%$ (345/385) of the time. A total of 57\% (220/385) of patients underwent an operation, but $11 \%(43 / 385)$ of patients who underwent an operation did not undergo resection due to locally advanced disease or occult metastatic disease. For the $46 \%$ (177/385) of patients who underwent neoadjuvant therapy and underwent resection, 91\% (161/177) of the had R0 resections (Figure 2). For the 385 patients who underwent neoadjuvant therapy with intent for resection, ultimately 42\% (161/385) of patients had an R0 margin after neoadjuvant therapy and $4 \%(16 / 385)$ of patients who underwent resection had positive margins (Figure 2).

Given that the survival for patients who undergo neoadjuvant therapy alone or followed by an operation without resection is approximately 8 months, while patients who undergo R0 resections is approximately 21 months and patients who undergo R1 or R2 resections is approximately 10 months, we estimated the theoretical survival per patient to be 13.5 months for patients undergoing neoadjuvant therapy prior to resection and 15.3 months for patients undergoing a "resection first" approach (Figure 3) $[5,18,19]$. We also estimated that about $25 \%$ of patients who undergo a "resection first" approach will have unresectable disease at the time of operation or have occult metastatic disease at the time of operation based on our institutional review (25\% nontherapeutic celiotomies) [20]. Additionally, patients who underwent staging laparoscopy for pancreatic adenocarcinoma have shown that staging laparoscopy could po-

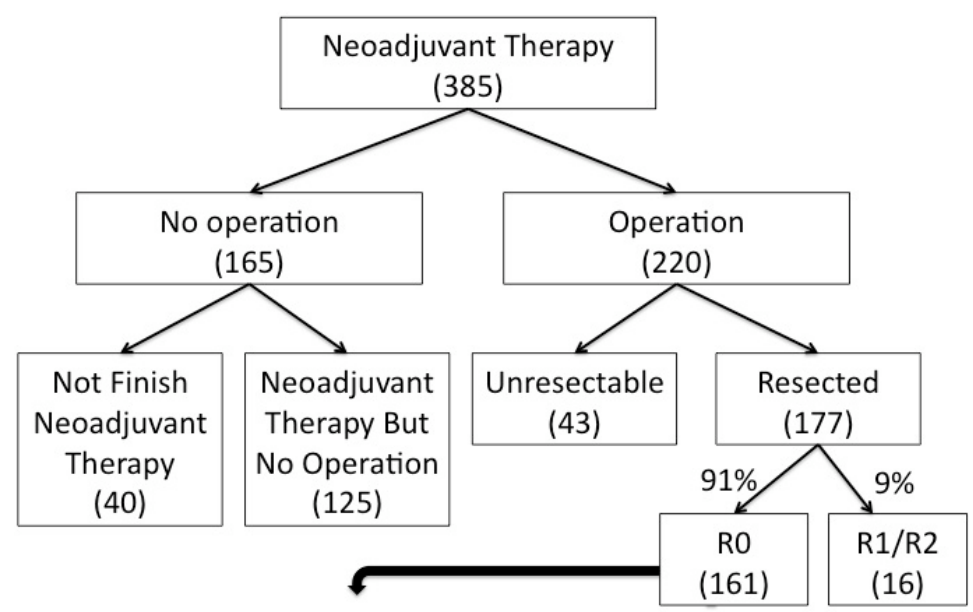

$161 / 385=42 \%$ of patients

Figure 2. Flowchart of outcomes for patients who underwent neoadjuvant therapy $(n=8$ studies with all pathways [12,23,24, $29,31,32,34,36]$ ) with ultimately $42 \%$ of patients obtaining an $\mathrm{RO}$ resection after neoadjuvant therapy. 

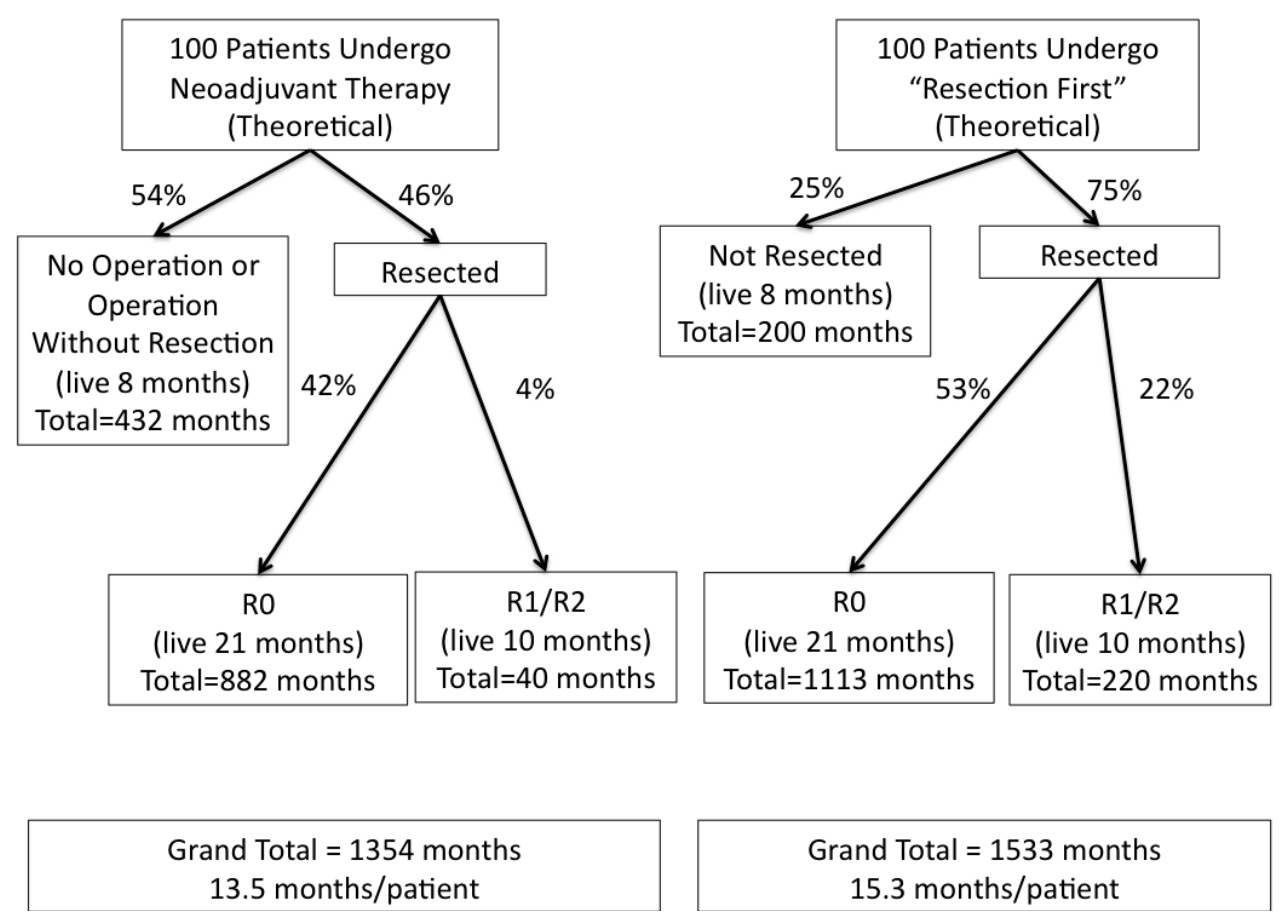

Figure 3. Flowchart of 100 patients who theoretically undergo neoadjuvant therapy prior to resection and 100 patients who theoretically undergo a "resection first" approach for borderline resectable pancreatic adenocarcinoma.

tentially avoid a nontherapeutic celiotomy $4 \%$ - 36\% of the time [21].

\section{Discussion}

Patients with pancreatic adenocarcinoma have a dismal prognosis. Resection continues to be the only hope of cure. Only about $20 \%$ of patients with pancreatic adenocarcinoma have disease that is potentially amenable to resection at the time of diagnosis. "Borderline resectable disease" was defined less than a decade ago and since its inception, it seems that the majority of patients with borderline resectable pancreatic adenocarcinoma have undergone neoadjuvant therapy. Additionally, now the standard chemotherapy for pancreatic adenocarcinoma, gemcitabine, has been shown to increase rates of tumor response, yet still with dismal results. A recent metaanalysis of all patients who underwent neoadjuvant gemcitabine therapy who were initially resectable, $12 \%$ had an objective tumor response, while $27 \%$ of patients with initially unresectable disease had an objective tumor response [16]. It is not well understood, however, if these objective responses translate into a salutary impact for patients as "tumor response" may be a consequence of true tumor regression or due to reduction in peritumoral inflammatory changes (i.e. peritumoral pancreatitis).

Less than half of the patients who underwent neoadjuvant therapy for borderline resectable pancreatic adenocarcinoma ultimately underwent resection. Patients who underwent upfront resection for borderline resectable pancreatic adenocarcinoma had an R0 resection margin over two-thirds of the time, while patients who underwent neoadjuvant therapy had an R0 resection margin less than half of the time. Patients who undergo resection after neoadjuvant therapy do have an impressive R0 resection rate, but events during neoadjuvant therapy prevent any potential resection for over half of the patients. Given these data, it appears that about one-third of patients would benefit from upfront resection instead of neoadjuvant therapy.

It is unknown, though, how patients with borderline resectable pancreatic adenocarcinoma will respond to neoadjuvant therapy. Logically, neoadjuvant therapy for borderline resectable pancreatic adenocarcinoma has been undertaken to increase the likelihood of resectability and negative margins at the time of resection. However, this systematic review concludes that neoadjuvant therapy dramatically decreases the chance of resection and, thereby, negative margins when compared to upfront resection (42\% of patients after neoadjuvant therapy have an $\mathrm{R} 0$ resection vs. $71 \%$ after a resection first approach). We estimate that adoption of the "resection first" approach would portend an increase in overall survival of about 1.8 months per patient (Figure 3). We feel the survival estimations are fair and objective, but each estimate could be debated.

The definitions for borderline resectable pancreatic 
Table 2. Studies that include patients who underwent neoadjuvant therapy for borderline resectable pancreatic adenocarcinoma; "Studies from same institution with overlapping time interval (Pimiento et al. included for analysis); 5FU (5-fluorouracil); Intensity-Modulated Radiation Therapy (IMRT); computer controlled radiation therapy (CCRT); Stereotactic Body Radiation Therapy (SBRT); XRT (external beam radiation therapy); RT (radiation therapy).

\begin{tabular}{|c|c|c|c|}
\hline Author last name & Regimen based & Concomitant chemotherapy & Radiation therapy \\
\hline Kang [24] & Gemcitabine & $+/$-Cisplatin & CCRT \\
\hline Pimiento [23] ${ }^{*}$ & Gemcitabine 5FU & $\begin{array}{c}\text { +/-Docetaxol/capecitabine } \\
\text { Erlotinib }\end{array}$ & $+/-5 F U / I M R T$ or SBRT \\
\hline Pipas [25] 2012 & Gemcitabine & Cetuximab & IMRT \\
\hline \multirow{10}{*}{ Arvold [26] } & & +/-Oxaliplatin & \\
\hline & & Capecitabine & \\
\hline & & Erlotinib & \\
\hline & Gemcitabine & Bevacizumab & \\
\hline & & Bevacizumab/erlotinib & \\
\hline & & Sunitinib & $+/-$ XRT \\
\hline & & Docetaxel & \\
\hline & 5FU/leucovorin & None & \\
\hline & Capecit & $+/-5 \mathrm{FU}$ & \\
\hline & $5 \mathrm{FU}$ & Other & \\
\hline Chuong [27] ${ }^{*}$ & Gemcitabine & Docetaxol/capecitabine & 5FU/IMRT \\
\hline Patel $[28]^{*}$ & Gemcitabine & Docetaxol/capecitabine & 5FU/IMRT \\
\hline Stokes [29] & Capecitabine & None & XRT \\
\hline Takahashi [30] & Chemotherapy & Unknown & $+/-\mathrm{RT}$ \\
\hline \multirow[t]{2}{*}{ McClaine [31] } & Gemcitabine & $\begin{array}{l}\text { +/-Erlotinib } \\
\text { +/-Oxaliplatin }\end{array}$ & +/-XRT/5FU or XRT/gemcitabine \\
\hline & Gemcitabine & $+/-5 \mathrm{FU}+\mathrm{XRT}$ & \\
\hline \multirow[t]{3}{*}{ Piperdi [32] } & Capecitabine & None & XRT \\
\hline & $5 \mathrm{FU}$ & None & \\
\hline & Gemcitabine & None & \\
\hline \multirow[t]{2}{*}{ Brown [33] } & $5 \mathrm{FU}$ & None & None \\
\hline & Capecitabine & Bevacizumab & \\
\hline Katz [12] & Gemcitabine & +/-Conconmitant chemotherapy & $\mathrm{XRT} / 5 \mathrm{FU}$ or XRT/gemcitabine or XRT/Cap or XRT/paclitaxel \\
\hline Marti [34] & Gemcitabine & Oxaliplatin & $+/-$ CCRT \\
\hline Small [35] & Gemcitabine & None & XRT \\
\hline Massucco [36] & Gemcitabine & +/-Oxaliplatin & +ChemoXRT \\
\hline Pipas [37] 2005 & Gemcitabine & Docetaxel & Gemcitabine/XRT \\
\hline
\end{tabular}

adenocarcinoma have evolved to be more encompassing. The most recent definition by the AHPBA/SSO/SSAT consensus now includes patients with portovenous abut- ment alone without impingement. The original definitions established by Varadhachary et al. in 2005 deemed patients with borderline resectable pancreatic adenocar 
Table 3. Number of patients who underwent neoadjuvant therapy, did not finish neoadjuvant therapy, finished neoadjuvant therapy but did not undergo an operation, underwent an operation after neoadjuvant therapy, and underwent resection after neoadjuvant therapy (n = 12 studies); ${ }^{*}$ Stokes includes 3 patients resected with borderline resectable pancreatic adenocarcinoma due to performance status ${ }^{* *}$ Katz Type B (borderline resectable pancreatic adenocarcinoma due to concern for extrapancreatic disease from CT or EUS/FNA findings or prior laparotomy with nodal disease; $n=44$ ) and Katz Type $C$ (borderline resectable pancreatic adenocarcinoma due to performance status; $\mathbf{n}=32$ ) included.

\begin{tabular}{|c|c|c|c|c|c|c|c|}
\hline Author last name & Year & Site & $\begin{array}{l}\text { Neoadjuvant } \\
\text { therapy }\end{array}$ & $\begin{array}{l}\text { Incomplete } \\
\text { neoadjuvant } \\
\text { therapy }\end{array}$ & $\begin{array}{l}\text { Finished neoadjuvant } \\
\text { therapy but did not } \\
\text { undergo an operation }\end{array}$ & $\begin{array}{c}\text { Operation after } \\
\text { neoadjuvant } \\
\text { therapy }\end{array}$ & $\begin{array}{c}\text { Resection after } \\
\text { neoadjuvant } \\
\text { therapy }\end{array}$ \\
\hline Kang [24] & 2012 & South Korea & 67 & $0(0 \%)$ & 35 (52\%) & 32 (48\%) & 32 (48\%) \\
\hline Pimiento [23] & 2012 & Moffitt & 60 & $8(13 \%)$ & $8(13 \%)$ & 44 (73\%) & 35 (58\%) \\
\hline Pipas [25] & 2012 & Multicenter & 23 & NA & NA & NA & 18 (78\%) \\
\hline Takahashi [30] & 2011 & Japan & 10 & NA & NA & NA & $2(20 \%)$ \\
\hline Stokes [29] ${ }^{*}$ & 2011 & UVA & 40 & $6(15 \%)$ & $12(30 \%)$ & 22 (55\%) & $16(40 \%)$ \\
\hline Arvold [26] & 2011 & Mass Gen & 24 & NA & NA & NA & $14(58 \%)$ \\
\hline Piperdi [32] & 2010 & UMass & 8 & $1(13 \%)$ & $0(0 \%)$ & $7(88 \%)$ & $6(75 \%)$ \\
\hline McClaine [31] & 2010 & Cincinnati & 29 & $3(10 \%)$ & $0(0 \%)$ & 26 (90\%) & 12 (41\%) \\
\hline Brown [33] & 2008 & $\begin{array}{c}\text { Fox Chase } \\
\text { (Philadelphia) }\end{array}$ & 13 & NA & NA & NA & 13 (100\%) \\
\hline Katz [12] & 2008 & MD Anderson & 160 & 21 (13\%) & 60 (38\%) & 79 (49\%) & $66(41 \%)$ \\
\hline Marti [34] & 2008 & NYU/Maimonides & 3 & $0(0 \%)$ & $0(0 \%)$ & 3 (100\%) & $3(100 \%)$ \\
\hline Small [35] & 2008 & Multicenter & 9 & NA & NA & NA & 3 (33\%) \\
\hline Massucco [36] & 2006 & Italy & 18 & $1(6 \%)$ & $10(56 \%)$ & $7(39 \%)$ & $7(39 \%)$ \\
\hline Pipas [37] & 2005 & $\begin{array}{l}\text { Dartmouth-Hitchcock } \\
\text { Medical Center/Norris } \\
\text { Cotton Cancer Center }\end{array}$ & 7 & NA & NA & NA & $6(86 \%)$ \\
\hline
\end{tabular}

Table 4. Outcomes for patients who underwent an operation after neoadjuvant therapy; *Stokes et al includes three patients resected with borderline resectable pancreatic adenocarcinoma due to performance status; ${ }^{* *}$ single patient assumed to be $\mathrm{R} 1$ but not explicitly reported; R0 (microscopically/macroscopically negative), R1 (microscopically positive/macroscopically negative), R2 (microscopically/macroscopically positive).

\begin{tabular}{ccccccc}
\hline Author last name & Operation after neoadjuvant therapy & Resection after neoadjuvant therapy & R0 & R1 & R2 & Operation without resection \\
\hline Kang [24] & 32 & 32 & 28 & 3 & 1 & 0 \\
Pimiento [23] & 44 & 35 & 34 & 1 & $0^{* *}$ & 9 \\
Stokes [29] & 22 & 16 & 14 & 2 & 0 & 6 \\
Piperdi [32] & 7 & 6 & 6 & 0 & 0 & 1 \\
McClaine [31] & 26 & 12 & 8 & 4 & 0 & 14 \\
Brown [33] & NA & 13 & 11 & 2 & 0 & 0 \\
Katz [12] & 79 & 66 & 62 & 4 & 0 \\
Marti [34] & 3 & 3 & 3 & 0 & 0 \\
Massucco [36] & 7 & 7 & 6 & 1 & 0 \\
Pipas [37] & NA & 6 & 5 & 1 & 0 \\
\hline
\end{tabular}

cinoma as having short segment portovenous occlusion, while abutment alone was not sufficient. Given that the most recent definitions for borderline resectable pancreatic adenocarcinoma will deem an ever-increasing amount 
Table 5. Patients with borderline resectable pancreatic adenocarcinoma who underwent upfront resection (n $=4$ studies).

\begin{tabular}{ccccccc}
\hline Author last name & Year & Site & Resected & R0 & R1 & R2 \\
\hline Kang [24] & 2012 & South Korea & 35 & 27 & 6 & 2 \\
Takahashi [30] & 2011 & Japan & 24 & 17 & 5 & 2 \\
Shrikhande [38] & 2011 & India & 7 & 5 & 5 & 0 \\
Piperdi [32] & 2010 & UMass & 10 & & 5 \\
\hline
\end{tabular}

of patients as having borderline resectable pancreatic adenocarcinoma, it is imperative to determine whether a neoadjuvant therapy or an upfront resection approach yields the most resectable disease with negative margins. Additionally, borderline resectable pancreatic adenocarcinoma as defined by Katz et al. includes patients patients with "borderline resectability" due to concern for extrapancreatic disease (Type B) or performance status (Type C) [12]. The inclusion of these additional patient samples, especially the patients with poor performance status as criteria for borderline resectable pancreatic adenocarcinoma confuses the data given these patients will never undergo resection given their comorbidities will continually preclude the patient from undergoing resection. Patients with comorbidities that preclude upfront resection should be considered candidates for chemoradiation alone as a primary therapy. We require a distinct and unified anatomical definition based on preoperative imaging.

Despite small, but incremental advances with chemotherapeutic strategies, neoadjuvant therapy for borderline resectable pancreatic adenocarcinoma portends a median survival equivalent to chemotherapy alone in over half of the patients. For these patients, neoadjuvant therapy was not neoadjuvant therapy, it was palliation because they never underwent resection. The studies available did have heterogeneity regarding chemotherapeutic regimens, but these are the available current data. Results for neoadjuvant therapy utilizing fluorouracil [5-FU], leucovorin, irinotecan and oxaliplatin (FOLFIRINOX) as well as gemcitabine with protein-bound paclitaxel (Abraxane $\left.{ }^{\circledR}\right)$ are anticipated, but are not yet available for patients with borderline resectable pancreatic adenocarcinoma.

Patients who present with borderline resectable pancreatic adenocarcinoma have two distinct options of neoadjuvant therapy or upfront resection. Given these data show that about one-third of patients would benefit from upfront resection to achieve R0 margins and more patients are being considered as having borderline resectable pancreatic adenocarcinoma, it is crucial that we undertake a multi-institutional randomized control trial comparing the dichotomized approaches. This manuscript cannot answer the questions as to which approach is best, only lay the groundwork for debate and foster a randomized control trial.

\section{Conclusion}

We expect that the paradigm will shift back to a resection first approach followed by adjuvant therapy, until more efficacious neoadjuvant therapies are established. In search of confirmation of more efficacious neoadjuvant therapies, we plan to undertake a trial for patients with initially resectable pancreatic adenocarcinoma using neoadjuvant gemcitabine with Abraxane, as the addition of protein-bound paclitaxel has been shown to induce superior tumor regression for patients with pancreatic adenocarcinoma as compared to gemcitabine-based therapy for patients with metastatic pancreatic adenocarcinoma [22].

\section{Acknowledgements}

We would like to thank Dr. Pimiento and Dr. Malafa as well as Dr. Chuong and Dr. Shridhar for allowing us access to their data prior to publication [23].

\section{REFERENCES}

[1] D. Henne-Bruns, I. Vogel, J. Luttges, et al., "Ductal Adenocarcinoma of the Pancreas Head: Survival after Regional versus Extended Lymphadenectomy,” Hepatogastroenterology, Vol. 45, No. 21, 1998, pp. 855-866.

[2] C. D. Johnson, G. Schwall, J. Flechtenmacher and M. Trede, "Resection for Adenocarcinoma of the Body and Tail of the Pancreas,” British Journal of Surgery, Vol. 80, No. 9, 1993, pp. 1177-1179. http://dx.doi.org/10.1002/bjs.1800800937

[3] M. Mukaiya, K. Hirata, T. Satoh, et al., "Lack of Survival Benefit of Extended Lymph Node Dissection for Ductal Adenocarcinoma of the Head of the Pancreas: Retrospective Multi-Institutional Analysis in Japan,” World Journal of Surgery, Vol. 22, No. 3, 1998, pp. 248-252. http://dx.doi.org/10.1007/s002689900378

[4] S. P. Cleary, R. Gryfe, M. Guindi, et al., "Prognostic Factors in Resected Pancreatic Adenocarcinoma: Analysis of Actual 5-Year Survivors,” Journal of the American College of Surgeons, Vol. 198, No. 5, 2004, pp. 722-731. http://dx.doi.org/10.1016/j.jamcollsurg.2004.01.008

[5] J. Hernandez, J. Mullinax, W. Clark, et al., "Survival after Pancreaticoduodenectomy Is Not Improved by Ex- 
tending Resections to Achieve Negative Margins," Annals of Surgery, Vol. 250, No. 1, 2009, pp. 76-80. http://dx.doi.org/10.1097/01.sla.0000259391.84304.2b

[6] C. P. Raut, J. F. Tseng, C. C. Sun, et al., "Impact of Resection Status on Pattern of Failure and Survival after Pancreaticoduodenectomy for Pancreatic Adenocarcinoma,” Annals of Surgery, Vol. 246, No. 1, 2007, pp. 52-60. http://dx.doi.org/10.1097/01.sla.0000259391.84304.2b

[7] P. Toomey, J. Hernandez, C. Morton, et al., "Resection of Portovenous Structures to Obtain Microscopically Negative Margins during Pancreaticoduodenectomy for Pancreatic Adenocarcinoma Is Worthwhile," American Journal of Surgery, Vol. 75, No. 9, 2009, pp. 804-809.

[8] J. F. Helm, B. A. Centeno, D. Coppola, et al., "Outcomes Following Resection of Pancreatic Adenocarcinoma: 20Year Experience at a Single Institution,” Cancer Control, Vol. 15, No. 4, 2008, pp. 288-294.

[9] P. Toomey, J. Hernandez, F. Golkar, et al., "Pancreatic Adenocarcinoma: Complete Tumor Extirpation Improves Survival Benefit despite Larger Tumors for Patients Who Undergo Distal Pancreatectomy and Splenectomy,” Journal of Gastrointestinal Surgery, Vol. 16, No. 2, 2012, pp. 376-381.

[10] G. R. Varadhachary, E. P. Tamm, J. L. Abbruzzese, et al., "Borderline Resectable Pancreatic Cancer: Definitions, Management, and Role of Preoperative Therapy,” Annals of Surgical Oncology, Vol. 13, No. 8, 2006, pp. 10351046. http://dx.doi.org/10.1245/ASO.2006.08.011

[11] G. R. Varadhachary, E. P. Tamm, C. Crane, et al., "Borderline Resectable Pancreatic Cancer," Current Treatment Options in Gastroenterology, Vol. 8, No. 5, 2005, pp. 377-384. http://dx.doi.org/10.1007/s11938-005-0040-x

[12] M. H. Katz, P. W. Pisters, D. B. Evans, et al., "Borderline Resectable Pancreatic Cancer: The Importance of This Emerging Stage of Disease," Journal of the American College of Surgeons, Vol. 206, No. 5, 2008, pp. 833-846. http://dx.doi.org/10.1016/j.jamcollsurg.2007.12.020

[13] M. P. Callery, K. J. Chang, E. K. Fishman, et al., "Pretreatment Assessment of Resectable and Borderline Resectable Pancreatic Cancer: Expert Consensus Statement," Annals of Surgical Oncology, Vol. 16, No. 7, 2009, pp. 1727-1733. http://dx.doi.org/10.1245/s10434-009-0408-6

[14] H. A. Burris III, M. J. Moore, J. Andersen, et al., "Improvements in Survival and Clinical Benefit with Gemcitabine as First-Line Therapy for Patients with Advanced Pancreas Cancer: A Randomized Trial,” Journal of Clinical Oncology, Vol. 15, No. 6, 1997, pp. 2403-2413.

[15] D. Tassinari, "Surrogate End Points of Quality of Life Assessment: Have We Really Found What We Are Looking for?" Health and Quality of Life Outcomes, Vol. 1, 2003, p. 71. http://dx.doi.org/10.1186/1477-7525-1-71

[16] A. Andriulli, V. Festa, E. Botteri, et al., "Neoadjuvant/ Preoperative Gemcitabine for Patients with Localized Pancreatic Cancer: A Meta-Analysis of Prospective Studies,” Annals of Surgical Oncology, Vol. 19, No. 5, 2012, pp. 1644-1662. http://dx.doi.org/10.1245/s10434-011-2110-8

[17] D. Habermehl, K. Kessel, T. Welzel, et al., "Neoadjuvant
Chemoradiation with Gemcitabine for Locally Advanced Pancreatic Cancer,” Radiation Oncology, Vol. 7, 2012, p. 28. http://dx.doi.org/10.1186/1748-717X-7-28

[18] S. Gillen, T. Schuster, C. Meyer Zum Buschenfelde, et al., "Preoperative/Neoadjuvant Therapy in Pancreatic Cancer: A Systematic Review and Meta-Analysis of Response and Resection Percentages," PLOS Medicine, Vol. 7, No. 4, 2010, Article ID: e1000267.

http://dx.doi.org/10.1371/journal.pmed.1000267

[19] C. J. Yeo, J. L. Cameron, K. D. Lillemoe, et al., "Pancreaticoduodenectomy for Cancer of the Head of the Pancreas. 201 Patients,” Annals of Surgery, Vol. 221, No. 6, 1995, pp. 721-731.

http://dx.doi.org/10.1097/00000658-199506000-00011

[20] P. Toomey, C. Childs, K. Luberice, S. Ross and A. Rosemurgy, "Nontherapeutic Celiotomy Incidence Is Not Affected by Volume of Pancreaticoduodenectomy for Pancreatic Adenocarcinoma," American Journal of Surgery, Vol. 79, No. 8, 2013, pp. 781-785.

[21] L. Chang, D. Stefanidis, W. S. Richardson, et al., "The Role of Staging Laparoscopy for Intraabdominal Cancers: An Evidence-Based Review," Surgical Endoscopy, Vol. 23, No. 2, 2009, pp. 231-241. http://dx.doi.org/10.1007/s00464-008-0099-2

[22] D. D. Von Hoff, R. K. Ramanathan, M. J. Borad, et al., "Gemcitabine plus Nab-Paclitaxel Is an Active Regimen in Patients with Advanced Pancreatic Cancer: A Phase I/II Trial,” Journal of Clinical Oncology, Vol. 29, No. 34, 2011, pp. 4548-4554. http://dx.doi.org/10.1200/JCO.2011.36.5742

[23] J. M. Pimiento, T. Hutchinson, J. M. Weber, M. R. Patel, P. J. Hodul, M. D. Chuong, G. M. Springett, S E. Hoffe, R. C. Karl, B. Centeno, J. Klapman, R. Shridhar, D. Chen and M. P. Malafa, "Multimodality Therapy for Borderline Resectable Pancreatic Cancer: A Single-Institution Experience,” Journal of Clinical Oncology, Vol. 30, Suppl. 4, 2012, Abstract 280.

[24] C. M. Kang, Y. E. Chung, J. Y. Park, et al., "Potential Contribution of Preoperative Neoadjuvant Concurrent Chemoradiation Therapy on Margin-Negative Resection in Borderline Resectable Pancreatic Cancer," Journal of Gastrointestinal Surgery, Vol. 16, No. 3, 2012, pp. 509517. http://dx.doi.org/10.1093/annonc/mds109

[25] J. M. Pipas, B. I. Zaki, M. M. McGowan, et al., "Neoadjuvant Cetuximab, Twice-Weekly Gemcitabine, and Intensity-Modulated Radiotherapy (IMRT) in Patients with Pancreatic Adenocarcinoma," Annals of Oncology, Vol. 23, No. 11, 2012, pp. 2820-2827.

http://dx.doi.org/10.1093/annonc/mds109

[26] N. D. Arvold, D. P. Ryan, A. Niemierko, et al., "LongTerm Outcomes of Neoadjuvant Chemotherapy before Chemoradiation for Locally Advanced Pancreatic Cancer,” Cancer, Vol. 118, No. 12, 2012, pp. 3026-3035.

[27] M. D. Chuong, T. J. Hayman, M. R. Patel, et al., "Comparison of 1-, 2-, and 3-Dimensional Tumor Response Assessment after Neoadjuvant GTX-RT in BorderlineResectable Pancreatic Cancer,” Gastrointestinal Cancer Research, Vol. 4, No. 4, 2011, pp. 128-134. 
[28] M. Patel, S. Hoffe, M. Malafa, et al., “Neoadjuvant GTX Chemotherapy and IMRT-Based Chemoradiation for Borderline Resectable Pancreatic Cancer,” Journal of Surgical Oncology, Vol. 104, No. 2, 2011, pp. 155-161. http://dx.doi.org/10.1002/jso.21954

[29] J. B. Stokes, N. J. Nolan, E. B. Stelow, et al., "Preoperative Capecitabine and Concurrent Radiation for Borderline Resectable Pancreatic Cancer," Annals of Surgical Oncology, Vol. 18, No. 3, 2011, pp. 619-627. http://dx.doi.org/10.1245/s10434-010-1456-7

[30] S. Takahashi, T. Kinoshita, M. Konishi, et al., "Borderline Resectable Pancreatic Cancer: Rationale for Multidisciplinary Treatment," Journal of Hepato-Biliary-Pancreatic Sciences, Vol. 18, No. 4, 2011, pp. 567-574. http://dx.doi.org/10.1007/s00534-011-0371-z

[31] R. J. McClaine, A. M. Lowy, J. J. Sussman, et al., "Neoadjuvant Therapy May Lead to Successful Surgical Resection and Improved Survival in Patients with Borderline Resectable Pancreatic Cancer,” HPB (Oxford), Vol. 12, No. 1, 2010, pp. 73-79. http://dx.doi.org/10.1111/j.1477-2574.2009.00136.x

[32] M. Piperdi, T. P. McDade, J. K. Shim, et al., “A Neoadjuvant Strategy for Pancreatic Adenocarcinoma Increases the Likelihood of Receiving All Components of Care: Lessons from a Single-Institution Database," $H P B(O x-$ ford), Vol. 12, No. 3, 2010, pp. 204-210.

[33] K. M. Brown, V. Siripurapu, M. Davidson, et al., "Chemoradiation Followed by Chemotherapy before Resection for Borderline Pancreatic Adenocarcinoma,” American Journal of Surgery, Vol. 195, No. 3, 2008, pp. 318-321. http://dx.doi.org/10.1016/j.amjsurg.2007.12.017

\section{Abbreviations}

BLR: borderline resectable

R0: microscopically/macroscopically negative

R1: microscopically positive/macroscopically negative

R2: microscopically/macroscopically positive

5FU: 5-fluorouracil
[34] J. L. Marti, H. S. Hochster, S. P. Hiotis, et al., "Phase I/II Trial of Induction Chemotherapy Followed by Concurrent Chemoradiotherapy and Surgery for Locoregionally Advanced Pancreatic Cancer,” Annals of Surgical Oncology, Vol. 15, No. 12, 2008, pp. 3521-3531. http://dx.doi.org/10.1245/s10434-008-0152-3

[35] W. Small Jr., J. Berlin, G. M. Freedman, et al., "FullDose Gemcitabine with Concurrent Radiation Therapy in Patients with Nonmetastatic Pancreatic Cancer: A Multicenter Phase II Trial,” Journal of Clinical Oncology, Vol. 26, No. 6, 2008, pp. 942-947. http://dx.doi.org/10.1200/JCO.2007.13.9014

[36] P. Massucco, L. Capussotti, A. Magnino, et al., "Pancreatic Resections after Chemoradiotherapy for Locally Advanced Ductal Adenocarcinoma: Analysis of Perioperative Outcome and Survival,” Annals of Surgical Oncology, Vol. 13, No. 9, 2006, pp. 1201-1208. http://dx.doi.org/10.1245/s10434-006-9032-x

[37] J. M. Pipas, R. J. Barth Jr., B. Zaki, et al., "Docetaxel/ Gemcitabine Followed by Gemcitabine and External Beam Radiotherapy in Patients with Pancreatic Adenocarcinoma," Annals of Surgical Oncology, Vol. 12, No. 12, 2005, pp. 995-1004. http://dx.doi.org/10.1245/ASO.2005.04.503

[38] S. V. Shrikhande, S. Arya, S. G. Barreto, et al., "Borderline Resectable Pancreatic Tumors: Is There a Need for Further Refinement of This Stage?" Hepatobiliary \& Pancreatic Diseases International, Vol. 10, No. 3, 2011, pp. 319-324.

http://dx.doi.org/10.1016/S1499-3872(11)60053-2

IMRT: intensity-modulated radiation therapy

CCRT: computer controlled radiation therapy

SBRT: stereotactic body radiation therapy

XRT: external beam radiation therapy

$\mathrm{RT}$ : radiation therapy 